\title{
Non-asymptotic fractional order differentiator for a class of fractional order linear systems *
}

\author{
Da-Yan Liu ${ }^{\mathrm{a}}$, Gang Zheng ${ }^{\mathrm{b}}$, Driss Boutat ${ }^{\mathrm{a}}$, Hao-Ran Liu ${ }^{\mathrm{c}}$ \\ ${ }^{a}$ INSA Centre Val de Loire, Université d'Orléans, PRISME EA 4229, Bourges Cedex 18022, France \\ ${ }^{\mathrm{b}}$ Non-A, INRIA-Lille Nord Europe, 40 avenue Halley, Villeneuve d'Ascq 59650, France. \\ ${ }^{\mathrm{c}}$ School of Information Science and Engineering, Yanshan University, Qinhuangdao, 066004, Hebei, China.
}

\begin{abstract}
This paper aims at designing a non-asymptotic fractional order differentiator for a class of fractional order linear systems to estimate the Riemann-Liouville fractional derivatives of the output in discrete noisy environment. The adopted method is a recent algebraic method originally introduced by Fliess and Sira-Ramirez. Firstly, the fractional derivative of the output of an arbitrary order is exactly given by a new algebraic formula in continuous noise free case without knowing the initial conditions of the considered system. Secondly, a digital fractional order differentiator is introduced in discrete noisy cases, which can provide robust estimations in finite-time. Then, some error analysis is given, where an error bound useful for the selection of the design parameter is provided. Finally, numerical examples illustrate the accuracy and the robustness of the proposed fractional order differentiator.
\end{abstract}

Key words: Fractional order linear systems, Riemann-Liouville fractional derivatives, Non-asymptotic fractional order differentiator, Algebraic method, Noise error analysis.

\section{Introduction}

Fractional calculus has a long history and has been becoming very useful in many scientific and engineering fields, including control, flow propagation, signal processing, electrical networks, etc. [1-6]. For instance, fractional order systems and controllers have been applied to improve performance and robustness properties in control design [7-9], where the fractional derivatives of the output usually need to be estimated from its discrete noisy observation. Consequently, an interesting research topic concerns with designing digital fractional order differentiators, which should be robust against noises.

Various robust fractional order differentiators have been proposed both in the frequency domain and in the time domain. They can be divided into two classes: model-

\footnotetext{
‡ This paper was not presented at any IFAC meeting. Corresponding author D. Y. Liu. Tel. +33-0248484091. Fax +330248484045 .

Email addresses: dayan.liu@insa-cvl.fr (Da-Yan Liu), gang.zheng@inria.fr (Gang Zheng), driss.boutat@insa-cvl.fr (Driss Boutat), liu.haoran@ysu.edu.cn (Hao-Ran Liu).
}

free fractional order differentiators [10-13] and modelbased ones [14-16]. The first class is obtained by truncating an analytical expression. Hence, this generates a truncated term error which can produce an amplitude error (in the vertical sense) and a shifted error (in the horizontal sense) [13]. The second class is obtained from the differential equations of considered signals. They do not introduce any truncated term errors.

Existing fractional order differentiators are usually extensions of integer order differentiators, which generally have one of the following disadvantages:

- they are sensible to noises, such as the well-known Grünwald-Letnikov scheme [5], which is the extension of the finite difference scheme and only efficient in noisefree case;

- they produce a truncated term error in the estimated derivatives, such as the model-free fractional order differentiators proposed in $[12,13]$, which are the extension of classical polynomial approximation methods;

- they asymmetrically converge, for instance, the fractional order Luenberger-like observer, considered as an model-based fractional order differentiator, is devoted to estimating the pseudo-state variables which are the 
fractional derivatives of the output [9]. Moreover, a fractional order observer cannot estimate the fractional derivatives of an arbitrary order.

Among the existing methods, there is a non-asymptotic algebraic method originally introduced by Fliess and Sira-Ramirez for linear identification [17]. This method permits to obtain exact algebraic integral formulae for the desired estimators, which can provide estimations in finite-time. It has been shown in [18] that, thanks to the integral formulae, these estimators exhibit good robustness properties with respect to corrupting noises. By considering these advantages, this method has been extended to design model-free integer order differentiators $[19,20]$ and model-based ones [21,22] for non-linear and linear integer order systems, respectively. More concretely, the latter ones estimate the integer order derivatives of the output of the following integer order linear system:

$$
\sum_{i=0}^{n} a_{i} y^{(i)}(t)=u(t),
$$

where $y$ and $u$ are the output and the input, respectively. Recently, these non-asymptotic and robust differentiators have been extended to fractional order case. In [13], a model-free differentiator has been designed without considering the system model. However, it produces a time-delay in the estimation. In [14], a model-based differentiator has been proposed to estimate the noninteger order derivatives of the output of the system defined in (1). For the same purpose, another model-based differentiator has been designed by applying the modulating function method in [15]. The latter two differentiators do not produce any time-delay in estimations. However, they are not applicable to fractional order systems. Having these ideas in mind, the objective of this paper is to extend the algebraic method to design a model-based differentiator to estimate the fractional derivatives of the output of the following fractional order linear system:

$$
\sum_{i=0}^{N} a_{i} \mathrm{D}_{t}^{\alpha_{i}} y(t)=\sum_{j=0}^{L} b_{j} \mathrm{D}_{t}^{\gamma_{j}} u(t)
$$

where $\mathrm{D}_{t}^{\alpha_{i}} y$ and $\mathrm{D}_{t}^{\gamma_{j}} u$ are the fractional derivatives of the output and the input, respectively.

The contributions of this paper can be outlined as follows:

- a digital model-based fractional order differentiator is introduced, which has the following advantages: $(i)$ it can be used to estimate the fractional derivative of the output of an arbitrary order; (ii) it is given by a new algebraic formula, which does not contain any source of errors in continuous noise free case; (iii) it can provide robust estimations in finite-time in discrete noisy cases, without any truncated term error;
- an error bound is provided for the selection of the design parameter;

- there is no need on the initial conditions of the considered system.

This paper is organized as follows: definitions and some useful properties of Riemann-Liouville fractional integrals and derivatives are recalled in Section 2. The main results are given in Section 3. Firstly, the algebraic method is applied to express the fractional derivatives of the output of the considered system by a new algebraic formula in continuous noise free case. Secondly, a digital fractional order differentiator is introduced in discrete noisy cases. Moreover, some error analysis is given. In Section 4, numerical results illustrate the accuracy and the robustness of the proposed fractional order differentiator. Finally, conclusions are summarized in Section 5.

\section{Preliminaries}

In this section, definitions and some useful properties of fractional integrals and derivatives are recalled. Moreover, some useful formulae related to the Laplace transform are given.

\subsection{Riemann-Liouville fractional integrals and deriva- tives}

Let $I=[0, h] \subset \mathbb{R}_{+}{ }^{1}, \alpha \in \mathbb{R}_{+}$, and $l=\lceil\alpha\rceil$, where $\lceil\alpha\rceil$ denotes the smallest integer larger than or equal to $\alpha$. Then, the following definitions are given.

Definition 1 ([1]p. 65) The $\alpha$ order Riemann-Liouville fractional integral of $f$ is defined on $] 0, h]$ as follows:

$$
\mathrm{D}_{t}^{-\alpha} f(t):= \begin{cases}f(t), & \text { if } \alpha=0, \\ \int_{0}^{t} \kappa_{\alpha}(t, \tau) f(\tau) d \tau, & \text { else }\end{cases}
$$

where $\kappa_{\alpha}(t, \cdot)$ is defined by:

$$
\kappa_{\alpha}(t, \tau):=\frac{1}{\Gamma(\alpha)}(t-\tau)^{\alpha-1}
$$

and $\Gamma(\cdot)$ is the well-known Gamma function.

Definition 2 ([1]p. 68) The $\alpha$ order Riemann-Liouville fractional derivative of $f$ is defined on $] 0, h]$ as follows:

$$
\mathrm{D}_{t}^{\alpha} f(t):=\frac{d^{l}}{d t^{l}}\left\{\mathrm{D}_{t}^{\alpha-l} f(t)\right\}
$$

\footnotetext{
1 In this paper, $\mathbb{R}_{+}$denotes the set of positive real numbers, $\mathbb{R}_{+}^{*}\left(\right.$ resp. $\left.\mathbb{N}^{*}\right)$ denotes the set of strictly positive real numbers (resp. strictly positive integers), and $\mathbb{Z}_{-}^{*}$ denotes the set of strictly negative integers.
} 
Remark 1 According to (3), if $0<\alpha<1$, the Riemann-Liouville fractional integrals are defined by improper integrals. Thus, if $l \neq \alpha$, the Riemann-Liouville fractional derivatives are also defined by improper integrals in (5).

The following formula establishes an additive index law for Riemann-Liouville fractional integrals and derivatives ([1] pp. 71-74): $\forall n \in \mathbb{N}, \alpha \in \mathbb{R}$,

$$
\frac{d^{n}}{d t^{n}}\left\{\mathrm{D}_{t}^{\alpha} f(t)\right\}=\mathrm{D}_{t}^{n+\alpha} f(t)
$$

In the following Lemma, the Leibniz formula for Riemann-Liouville integrals involving a polynomial is recalled. The general Leibniz formula for RiemannLiouville integrals and derivatives can be found in [2] (p. 75) and [4] (p. 33), respectively.

Lemma 1 [2] (p. 53) Let $\alpha \in \mathbb{R}_{+}^{*}$ and $m \in \mathbb{N}$. Then, the following formula holds:

$\mathrm{D}_{t}^{-\alpha}\left[t^{m} f(t)\right]=\sum_{k=0}^{m}(-1)^{k}\left(\begin{array}{c}m \\ k\end{array}\right) \frac{\Gamma(\alpha+k)}{\Gamma(\alpha)} t^{m-k} \mathrm{D}_{t}^{-\alpha-k} f(t)$.

\subsection{Laplace transform formulae}

Let us assume that the Laplace transform of $f$ exists, which is denoted by $\hat{f}$. Then, the Laplace transforms of the Riemann-Liouville integrals and derivatives of $f$ are respectively given on $\mathbb{C}$ by ([3] p. 284): $\forall \alpha \in \mathbb{R}_{+}^{*}$,

$$
\begin{aligned}
\mathcal{L}\left\{\mathrm{D}_{t}^{-\alpha} f(t)\right\}(s) & =\frac{1}{s^{\alpha}} \hat{f}(s), \\
\mathcal{L}\left\{\mathrm{D}_{t}^{\alpha} f(t)\right\}(s) & =s^{\alpha} \hat{f}(s)-\sum_{j=0}^{\lceil\alpha\rceil-1} s^{j}\left[\mathrm{D}_{t}^{\alpha-j-1} f(t)\right]_{t=0},
\end{aligned}
$$

where $s$ denotes the variable in the frequency domain. Then, the following lemma can be obtained.

Lemma 2 [14] Let $\alpha \in \mathbb{R}_{+}$and $n \in \mathbb{N}$. Then, the following formula holds:

$$
\mathcal{L}^{-1}\left\{\frac{1}{s^{\alpha}} \hat{f}^{(n)}(s)\right\}(t)=(-1)^{n} \mathrm{D}_{t}^{-\alpha}\left[t^{n} f(t)\right] .
$$

Finally, this section ends by giving the following formula: $\forall k \in \mathbb{N}^{*}, \alpha \in \mathbb{R}$,

$$
\frac{d^{k}}{d s^{k}}\left\{s^{\alpha}\right\}=\left\{\begin{array}{cl}
0, & \text { if } \alpha \in \mathbb{N} \text { and } \alpha<k, \\
(-1)^{k} \frac{(k-\alpha-1) !}{(-\alpha-1) !} s^{\alpha-k}, & \text { if } \alpha \in \mathbb{Z}_{-}^{*}, \\
\frac{\Gamma(\alpha+1)}{\Gamma(\alpha+1-k)} s^{\alpha-k}, & \text { else. }
\end{array}\right.
$$

\section{Main results}

\subsection{Problem formulation}

Within this framework, a class of fractional order linear systems are considered, which can be described by the following equation ([5], pp. 17-18):

$$
\sum_{i=0}^{N} a_{i} \mathrm{D}_{t}^{\alpha_{i}} y(t)=\sum_{j=0}^{L} b_{j} \mathrm{D}_{t}^{\gamma_{j}} u(t)
$$

on $I=[0, h] \subset \mathbb{R}_{+}$, where $y \in \mathbb{R}$ is the output, $u \in$ $\mathbb{R}$ is the input, $N \in \mathbb{N}^{*}, a_{N} \in \mathbb{R}^{*}, a_{i} \in \mathbb{R}$, for $i=$ $0, \ldots, N-1, \alpha_{i}=i \alpha$ for $i=0, \ldots, N, L \in \mathbb{N}, b_{j} \in \mathbb{R}$, $\gamma_{j}=j \alpha$ for $j=0, \ldots, L, \alpha=\frac{p}{q}, p, q \in \mathbb{N}^{*}$. In order to guarantee the stability of the considered fractional order systems, it is assumed that $0<\alpha<2$ [8]. Without loss of generality, $p$ is assumed to be equal to 1 in the sequel. Moreover, $u$ is assumed to be known. Thus, $\mathrm{D}_{t}^{\gamma_{j}} u$ can be analytical or numerically calculated. In order to simplify the presentation, it is assumed that $b_{0}=1$ and $b_{j}=0$ for $j=1, \ldots, L$.

For the systems studied in (12), when dealing with the problem of output regulation (stabilization of the output for example), normally the controller $u$ requires the fractional derivatives of $y[6]$. Sometimes, it prefers nonasymptotic one since it might provide estimations in finite-time. This motivates the research work of this paper on non-asymptotic fractional order differentiator. It should be noticed that this paper does not adopt the conventional state-space equation, but uses the inputoutput equation (12) to describe the fractional order linear systems. This choice is naturally due to the fact that the input-output representation is much more suitable by using the algebraic method to realize the desired nonasymptotic fractional order differentiator.

\subsection{Non-asymptotic fractional order differentiator}

As mentioned in Remark 1, the Riemann-Liouville fractional derivatives of $y$ and its Riemann-Liouville fractional integrals of order strictly smaller than 1 are defined by improper integrals. In order to overcome this problem, the algebraic method is applied in this subsection to express the latter by new algebraic formulae in continuous noise free case. These formulae involve proper integrals of $y$, which can be considered as a low-pass filter in noisy cases [18].

Firstly, the fractional integrals of order strictly smaller than 1 are studied in the following theorem. The idea is to apply the algebraic method to transform the fractional order differential equation (12) into a fractional order integral equation by eliminating the unknown initial conditions. For this purpose, the following notations are 
introduced: $\forall \beta_{d} \in\left[0,1\left[, \exists \beta_{q} \in\left[0, \frac{1}{q}[\right.\right.\right.$ and $d \in\{1, \ldots, q\}$, such that $\beta_{d}=1-\frac{d}{q}+\beta_{q}$.

Theorem 1 Lety be the output of a fractional order linear system defined by (12). Then, the $\beta_{d}$ order RiemannLiouville fractional integral of $y$ can be given on $] 0, h]$ by a recursive way as follows:

$$
\begin{aligned}
& \mathrm{D}_{t}^{-\beta_{d}} y(t)=-\sum_{i=0}^{N-1} \frac{a_{i}}{a_{N}} \mathrm{D}_{t}^{-\beta_{i, d}} y(t) \\
& +m_{0} \int_{0}^{t} P_{d, m}(t, \tau) y(\tau) d \tau+\int_{0}^{t} Q_{d, m}(t, \tau) u(\tau) d \tau,
\end{aligned}
$$

where $\beta_{i, d}=\alpha_{N}-\alpha_{i}+\beta_{d}=\frac{N-i}{q}+\beta_{d}, m \in \mathbb{N}^{*}$, satisfying $\left\lceil\alpha_{N}\right\rceil \leq m$, is a design parameter, $m_{0}=0$ if $m=0$, and $m_{0}=1$ else,

$$
\begin{aligned}
& P_{d, m}(t, \tau)= \\
& -\sum_{i=0}^{N_{\beta_{d}}} \frac{a_{i}}{a_{N}} \sum_{k=1}^{m}(-1)^{k}\left(\begin{array}{c}
m \\
k
\end{array}\right) \frac{\Gamma\left(\beta_{i, d}+k\right)}{\Gamma\left(\beta_{i, d}\right)} \frac{\kappa_{\beta_{i, d}+k}(t, \tau)}{t^{k}} \\
& -\sum_{i=0}^{N} \frac{a_{i}}{a_{N}} \sum_{j=1}^{m}\left(\begin{array}{c}
m \\
j
\end{array}\right) c_{i, j} \times \\
& \sum_{k=0}^{m-j}\left(\begin{array}{c}
m-j \\
k
\end{array}\right) \frac{\Gamma\left(\beta_{i, d}+j+k\right)}{\Gamma\left(\beta_{i, d}+j\right)} \frac{(-1)^{(j+k)}}{t^{j+k}} \kappa_{\beta_{i, d}+j+k}(t, \tau),
\end{aligned}
$$

and

$Q_{d, m}(t, \tau)=\frac{1}{a_{N}} \sum_{k=0}^{m}(-1)^{k}\left(\begin{array}{c}m \\ k\end{array}\right) \frac{\Gamma\left(\beta_{0, d}+k\right)}{\Gamma\left(\beta_{0, d}\right)} \frac{\kappa_{\beta_{0, d}+k}(t, \tau)}{t^{k}}$,

$N_{\beta_{d}}=N-1$ if $\beta_{d}=0, N_{\beta_{d}}=N$ else, $c_{i, j}$ are the coefficients of $\left(s^{\alpha_{i}}\right)^{(j)}$, which are given by (11), and $\kappa_{\beta_{i, d}+j+k}(t, \tau)$ can be given by (4).

Proof. In this proof, the algebraic method will be applied, where the following steps are required.

- Step 1. Laplace transform: By applying the Laplace transform to (12) and using (9), we get:

$\sum_{i=0}^{N} a_{i} s^{\alpha_{i}} \hat{y}(s)-\sum_{i=1}^{N} a_{i} \sum_{j=0}^{\left\lceil\alpha_{i}\right\rceil-1} s^{j}\left[\mathrm{D}_{t}^{\alpha_{i}-j-1} y(t)\right]_{t=0}=\hat{u}(s)$,

where $\hat{y}$ (resp. $\hat{u}$ ) denotes the Laplace transform of $\bar{y}$ (resp. $\bar{u}$ ), and $\bar{y}$ (resp. $\bar{u}$ ) is defined on $\mathbb{R}$ in such a way that $\bar{y}(t)=y(t)(\operatorname{resp} \cdot \bar{u}(t)=u(t))$ if $t \in I$, and $\bar{y}(t)=0$ (resp. $\bar{u}(t)=0)$, else.
- Step 2. Elimination of initial conditions: Since $0 \leq \alpha_{0}<\alpha_{1} \cdots<\alpha_{N}$, if $\left\lceil\alpha_{N}\right\rceil \leq m$, applying $m$ times differentiation with respect to $s$ allows us to eliminate the unknown initial conditions in (16):

$$
\hat{u}^{(m)}(s)=\sum_{i=0}^{N} a_{i} \frac{d^{m}}{d s^{m}}\left\{s^{\alpha_{i}} \hat{y}(s)\right\} .
$$

Then, by applying the classical Leibniz formula we get:

$$
\begin{aligned}
\hat{u}^{(m)}(s) & =\sum_{i=0}^{N} a_{i} s^{\alpha_{i}} \hat{y}^{(m)}(s) \\
+ & m_{0} \sum_{i=0}^{N} a_{i} \sum_{j=1}^{m}\left(\begin{array}{c}
m \\
j
\end{array}\right) c_{i, j} s^{\alpha_{i}-j} \hat{y}^{(m-j)}(s),
\end{aligned}
$$

where the coefficients $c_{i, j}$ of $\left(s^{\alpha_{i}}\right)^{(j)}$ are given by (11), $m_{0}=0$ if $m=0$, and $m_{0}=1$ else.

- Step 3. Division by $s^{\alpha_{N}+\beta}$ with $0 \leq \beta<1$ : In order to apply Lemma 2 to obtain an integral formula when returning back into the time domain, the powers of $s$ should become strictly negative in (18). Hence, dividing (18) by $s^{\alpha_{N}+\beta}$ gives:

$$
\begin{aligned}
& \frac{\hat{u}^{(m)}(s)}{s^{\alpha_{N}+\beta}}=\sum_{i=0}^{N} a_{i} \frac{\hat{y}^{(m)}(s)}{s^{\alpha_{N}-\alpha_{i}+\beta}} \\
&+m_{0} \sum_{i=0}^{N} a_{i} \sum_{j=1}^{m}\left(\begin{array}{c}
m \\
j
\end{array}\right) c_{i, j} \frac{\hat{y}^{(m-j)}(s)}{s^{\alpha_{N}-\alpha_{i}+\beta+j}} .
\end{aligned}
$$

- Step 4. Inverse of Laplace transform: When returning back into the time domain by applying the inverse of the Laplace transform, Lemma 2 gives: $\forall t \in$ ] $0, h]$,

$$
\begin{aligned}
& \sum_{i=0}^{N} a_{i} \mathrm{D}_{t}^{\alpha_{i}-\alpha_{N}-\beta}\left[t^{m} y(t)\right]=\mathrm{D}_{t}^{-\alpha_{N}-\beta}\left[t^{m} u(t)\right] \\
& -m_{0} \sum_{i=0}^{N} a_{i} \sum_{j=1}^{m}(-1)^{j}\left(\begin{array}{c}
m \\
j
\end{array}\right) c_{i, j} \mathrm{D}_{t}^{\alpha_{i}-\alpha_{N}-\beta-j}\left[t^{m-j} y(t)\right] .
\end{aligned}
$$

Thus, the differential equation (12) is transformed into an integral equation in (20) without the knowledge on the initial conditions.

- Step 5. Leibniz formula for fractional integrals: By applying Lemma 1 to the two sides of (20), we obtain: 
on the one hand,

$$
\begin{aligned}
& \sum_{i=0}^{N} a_{i} \mathrm{D}_{t}^{\alpha_{i}-\alpha_{N}-\beta}\left[t^{m} y(t)\right]= \\
& \sum_{k=0}^{m}(-1)^{k}\left(\begin{array}{c}
m \\
k
\end{array}\right) \frac{\Gamma\left(\alpha_{N}+\beta+k\right)}{\Gamma\left(\alpha_{N}+\beta\right)} t^{m-k} \mathrm{D}_{t}^{-\alpha_{N}-\beta-k} u(t) \\
& -m_{0} \sum_{i=0}^{N} a_{i} \sum_{j=1}^{m}\left(\begin{array}{c}
m \\
j
\end{array}\right) c_{i, j} \sum_{k=0}^{m-j}(-1)^{(k+j)}\left(\begin{array}{c}
m-j \\
k
\end{array}\right) \times \\
& \frac{\Gamma\left(\alpha_{N}-\alpha_{i}+\beta+j+k\right)}{\Gamma\left(\alpha_{N}-\alpha_{i}+\beta+j\right)} t^{m-j-k} \mathrm{D}_{t}^{\alpha_{i}-\alpha_{N}-\beta-j-k} y(t),
\end{aligned}
$$

on the other hand, if $0<\beta<1$, we have:

$$
\begin{aligned}
& \sum_{i=0}^{N} a_{i} \mathrm{D}_{t}^{\alpha_{i}-\alpha_{N}-\beta}\left[t^{m} y(t)\right]=\sum_{i=0}^{N} a_{i} \times \\
& \sum_{k=0}^{m}(-1)^{k}\left(\begin{array}{c}
m \\
k
\end{array}\right) \frac{\Gamma\left(\alpha_{N}-\alpha_{i}+\beta+k\right)}{\Gamma\left(\alpha_{N}-\alpha_{i}+\beta\right)} t^{m-k} \mathrm{D}_{t}^{\alpha_{i}-\alpha_{N}-\beta-k} y(t),
\end{aligned}
$$

and if $\beta=0$, we have:

$$
\begin{aligned}
& \sum_{i=0}^{N} a_{i} \mathrm{D}_{t}^{\alpha_{i}-\alpha_{N}-\beta}\left[t^{m} y(t)\right]=a_{N} t^{m} y(t)+\sum_{i=0}^{N-1} a_{i} \times \\
& \sum_{k=0}^{m}(-1)^{k}\left(\begin{array}{c}
m \\
k
\end{array}\right) \frac{\Gamma\left(\alpha_{N}-\alpha_{i}+k\right)}{\Gamma\left(\alpha_{N}-\alpha_{i}\right)} t^{m-k} \mathrm{D}_{t}^{\alpha_{i}-\alpha_{N}-k} y(t) .
\end{aligned}
$$

By regrouping the terms in the double sums in the right sides of (22) and (23), respectively, the following equation is obtained:

$$
\begin{aligned}
& \sum_{i=0}^{N} a_{i} \mathrm{D}_{t}^{\alpha_{i}-\alpha_{N}-\beta}\left[t^{m} y(t)\right]= \\
& \sum_{i=0}^{N} a_{i} t^{m} \mathrm{D}_{t}^{\alpha_{i}-\alpha_{N}-\beta} y(t)+m_{0} \sum_{i=0}^{N_{\beta}} a_{i} \sum_{k=1}^{m}(-1)^{k}\left(\begin{array}{c}
m \\
k
\end{array}\right) \times \\
& \frac{\Gamma\left(\alpha_{N}-\alpha_{i}+\beta+k\right)}{\Gamma\left(\alpha_{N}-\alpha_{i}+\beta\right)} t^{m-k} \mathrm{D}_{t}^{\alpha_{i}-\alpha_{N}-\beta-k} y(t),
\end{aligned}
$$

where $N_{\beta}=N$ if $0<\beta<1$, and $N_{\beta}=N-1$ if $\beta=0$.
Hence, using (24), (21) becomes:

$$
\begin{aligned}
& \sum_{i=0}^{N} a_{i} \mathrm{D}_{t}^{\alpha_{i}-\alpha_{N}-\beta} y(t)= \\
& \sum_{k=0}^{m}(-1)^{k}\left(\begin{array}{c}
m \\
k
\end{array}\right) \frac{\Gamma\left(\alpha_{N}+\beta+k\right)}{\Gamma\left(\alpha_{N}+\beta\right)} \frac{1}{t^{k}} \mathrm{D}_{t}^{-\alpha_{N}-\beta-k} u(t) \\
& -m_{0} \sum_{i=0}^{N_{\beta}} a_{i} \sum_{k=1}^{m}\left(\begin{array}{c}
m \\
k
\end{array}\right) \frac{\Gamma\left(\alpha_{N}-\alpha_{i}+\beta+k\right)}{\Gamma\left(\alpha_{N}-\alpha_{i}+\beta\right)} \frac{(-1)^{k}}{t^{k}} \mathrm{D}_{t}^{\alpha_{i}-\alpha_{N}-\beta-k} y(t) \\
& -m_{0} \sum_{i=0}^{N} a_{i} \sum_{j=1}^{m}\left(\begin{array}{c}
m \\
j
\end{array}\right) c_{i, j} \sum_{k=0}^{m-j}(-1)^{(k+j)}\left(\begin{array}{c}
m-j \\
k
\end{array}\right) \times \\
& \frac{\Gamma\left(\alpha_{N}-\alpha_{i}+\beta+j+k\right)}{\Gamma\left(\alpha_{N}-\alpha_{i}+\beta+j\right)} \frac{1}{t^{j+k}} \mathrm{D}_{t}^{\alpha_{i}-\alpha_{N}-\beta-j-k} y(t) .
\end{aligned}
$$

- Step 6. Recursive algorithm: Recall that $\alpha_{N}-\alpha_{i}+$ $\beta \overline{=\frac{N-i}{q}+\beta \text {, for } i=0, \ldots, N \text {. If } \beta}=\beta_{d}=1-\frac{d}{q}+\beta_{q}$ with $0 \leq \beta_{q}<\frac{1}{q}$, for $d=1, \ldots, q$, we get $\alpha_{N}-\alpha_{i}+\beta=\beta_{i, d}$ with $\beta_{i, d}=\frac{N-i-d}{q}+1+\beta_{q}$. Thus, according to (25), it yields:

$$
\begin{aligned}
& \mathrm{D}_{t}^{-\beta_{d}} y(t)=-\sum_{i=0}^{N-1} \frac{a_{i}}{a_{N}} \mathrm{D}_{t}^{-\beta_{i, d}} y(t) \\
& +\frac{1}{a_{N}} \sum_{k=0}^{m}(-1)^{k}\left(\begin{array}{c}
m \\
k
\end{array}\right) \frac{\Gamma\left(\beta_{0, d}+k\right)}{\Gamma\left(\beta_{0, d}\right)} \frac{1}{t^{k}} \mathrm{D}_{t}^{-\beta_{0, d}-k} u(t) \\
& -m_{0} \sum_{i=0}^{N_{\beta_{d}}} \frac{a_{i}}{a_{N}} \sum_{k=1}^{m}(-1)^{k}\left(\begin{array}{c}
m \\
k
\end{array}\right) \frac{\Gamma\left(\beta_{i, d}+k\right)}{\Gamma\left(\beta_{i, d}\right)} \frac{1}{t^{k}} \mathrm{D}_{t}^{-\beta_{i, d}-k} y(t) \\
& -m_{0} \sum_{i=0}^{N} \frac{a_{i}}{a_{N}} \sum_{j=1}^{m}\left(\begin{array}{c}
m \\
j
\end{array}\right) c_{i, j} \sum_{k=0}^{m-j}(-1)^{(j+k)}\left(\begin{array}{c}
m-j \\
k
\end{array}\right) \times \\
& \frac{\Gamma\left(\beta_{i, d}+j+k\right)}{\Gamma\left(\beta_{i, d}+j\right)} \frac{1}{t^{j+k}} \mathrm{D}_{t}^{-\beta_{i, d}-j-k} y(t),
\end{aligned}
$$

where $N_{\beta_{d}}=N-1$ if $\beta_{d}=0$, and $N_{\beta_{d}}=N$ else. Consequently, this proof is completed.

Remark 2 It can be seen in the proof of Theorem 1 that the $m$ times differentiation is devoted to eliminating the unknown initial conditions with $\left\lceil\alpha_{N}\right\rceil \leq m$. Hence, if the initial conditions are equal to zero, $m$ can be set to any positive integer number. In the next subsection, an error bound depending on $m$ will be provided in discrete noisy cases, then the role of the design parameter $m$ will be studied in the part of numerical simulations.

Thanks to Theorem $1, \forall \beta_{d} \in\left[0,1\left[, \mathrm{D}_{t}^{-\beta_{d}} y\right.\right.$ is exactly given by proper integrals of $y$ and integrals of $u$ using 
a recursive way. Indeed, in order to calculate $\mathrm{D}_{t}^{-\beta_{d}} y$ by applying (13)-(15), the required orders of the fractional integrals of $u$ and $y$ are given in Tab. 1 and Tab. 2, respectively, for $d=1, \ldots, q$, where we have:

- for $d=1, \mathrm{D}_{t}^{-\beta_{1}} y$ is given by the fractional integrals of $y$ (resp. of $u$ ) with orders varying from $1+\beta_{q}$ to $\alpha_{N}+\beta_{1}+m$ (resp. from $\alpha_{N}+\beta_{1}$ to $\alpha_{N}+\beta_{1}+m$ ), which are proper integrals;

- for $d=2, \ldots, q, \mathrm{D}_{t}^{-\beta_{d}} y$ is given by $\mathrm{D}_{t}^{-\beta_{b}} y$ for $b=$ $1, \ldots, d-1$, which are previously given by proper integrals, and by the fractional integrals of $y$ (resp. of $u$ ) with orders varying from $1+\beta_{q}$ to $\alpha_{N}+\beta_{d}+m$ (resp. from $\alpha_{N}+\beta_{d}$ to $\alpha_{N}+\beta_{d}+m$ ), which are proper integrals (resp. proper integrals if $\alpha_{N}+\beta_{d} \geq 1$, i.e. $N \geq d)$.

\begin{tabular}{|c|c|}
\hline \hline $\mathrm{D}_{t}^{-\beta_{d}} y$ & $\mathrm{D}_{t}^{-\beta_{0, d}-k} u$ \\
\hline$\beta_{1} \in\left[1-\frac{1}{q}, 1[\right.$ & $\left\{\alpha_{N}+\beta_{1}, \ldots, \alpha_{N}+\beta_{1}+m\right\}$ \\
\hline$\vdots$ & $\vdots$ \\
\hline$\beta_{d} \in\left[1-\frac{d}{q}, 1-\frac{d+1}{q}[\right.$ & $\left\{\alpha_{N}+\beta_{d}, \ldots, \alpha_{N}+\beta_{d}+m\right\}$ \\
\hline$\vdots$ & $\vdots$ \\
\hline$\beta_{q} \in\left[0, \frac{1}{q}[\right.$ & $\left\{\alpha_{N}+\beta_{q}, \ldots, \alpha_{N}+\beta_{q}+m\right\}$ \\
\hline \hline
\end{tabular}

Table 1

Required orders of the fractional integrals of $u$ in the formula of $\mathrm{D}_{t}^{-\beta_{d}} y$.

\begin{tabular}{|c|c|}
\hline \hline $\mathrm{D}_{t}^{-\beta_{d}} y$ & $\mathrm{D}_{t}^{-\beta_{i, d}} y$ \\
\hline$\beta_{1}$ & $\left\{\alpha_{1}+\beta_{1}=1+\beta_{q}, \ldots, \alpha_{N}+\beta_{1}\right\}$ \\
\hline$\vdots$ & $\vdots$ \\
\hline$\beta_{d}$ & $\left\{\alpha_{1}+\beta_{d}, \ldots, \beta_{1}, 1+\beta_{q}, \ldots, \alpha_{N}+\beta_{d}\right\}$ \\
\hline$\vdots$ & $\vdots$ \\
\hline$\beta_{q}$ & $\left\{\alpha_{1}+\beta_{q}, \ldots, \beta_{1}, 1+\beta_{q}, \ldots, \alpha_{N}+\beta_{q}\right\}$ \\
\hline $\mathrm{D}_{t}^{-\beta_{d}} y$ & $\mathrm{D}_{t}^{-\beta_{i, d}-k} y$ and $\mathrm{D}_{t}^{-\beta_{i, d}-j-k} y$ \\
\hline$\beta_{1}$ & $\left\{\alpha_{1}+\beta_{1}+1, \ldots, \alpha_{N}+\beta_{1}+m\right\}$ \\
\hline$\vdots$ & $\vdots$ \\
\hline$\beta_{d}$ & $\left\{\alpha_{1}+\beta_{d}+1, \ldots, \alpha_{N}+\beta_{d}+m\right\}$ \\
\hline$\vdots$ & $\vdots$ \\
\hline$\beta_{q}$ & $\left\{\alpha_{1}+\beta_{q}+1, \ldots, \alpha_{N}+\beta_{q}+m\right\}$ \\
\hline \hline
\end{tabular}

Table 2

Required orders of the fractional integrals of $y$ in the formula of $\mathrm{D}_{t}^{-\beta_{d}} y$.

According to (5), the Riemann-Liouville fractional derivatives of $y$ are defined by taking the integer order derivatives of the Riemann-Liouville fractional integrals of $y$ of order strictly smaller than 1. Inspired by this idea, the following corollary can be obtained using Theorem 1. Before doing so, the following notations are introduced: $\forall \nu_{d} \in \mathbb{R}_{+}^{*}, \exists \beta_{q} \in\left[0, \frac{1}{q}[\right.$ and $d \in\{1, \ldots, q\}$, such that $\nu_{d}=l-\beta_{d}$ with $l=\left\lceil\nu_{d}\right\rceil$ and $\beta_{d}=1-\frac{d}{q}+\beta_{q} \in[0,1[$. Hence, $\forall d \in\{1, \ldots, q\}$, we have: $l=\left\lceil\nu_{d}\right\rceil$, i.e. $l$ is independent of the index $d$.

Corollary 1 Lety be the output of a fractional order linear system defined by (12). Then, the $\nu_{d}$ order RiemannLiouville derivative of $y$ can be given on $] 0, h]$ by a recursive way as follows:

$$
\begin{aligned}
\mathrm{D}_{t}^{\nu_{d}} y(t)= & \frac{d^{l}}{d t^{l}}\left\{\mathrm{D}_{t}^{-\beta_{d}} y(t)\right\} \\
=- & \sum_{i=0}^{N-1} \frac{a_{i}}{a_{N}} \mathrm{D}_{t}^{l-\beta_{i, d}} y(t)+m_{0} \frac{d^{l}}{d t^{l}} \int_{0}^{t} P_{d, m}(t, \tau) y(\tau) d \tau \\
& +\frac{d^{l}}{d t^{l}} \int_{0}^{t} Q_{d, m}(t, \tau) u(\tau) d \tau
\end{aligned}
$$

where $\beta_{i, d}=\alpha_{N}-\alpha_{i}+\beta_{d}, m_{0}=0$ if $m=0$, and $m_{0}=1$ else,

$$
\begin{aligned}
& \frac{d^{l}}{d t^{l}} \int_{0}^{t} P_{d, m}(t, \tau) y(\tau) d \tau=-\sum_{i=0}^{N_{\beta_{d}}} \frac{a_{i}}{a_{N}} \sum_{k=1}^{m}\left(\begin{array}{c}
m \\
k
\end{array}\right) \frac{\Gamma\left(\beta_{i, d}+k\right)}{\Gamma\left(\beta_{i, d}\right)} \times \\
& \left(\sum_{k^{\prime}=0}^{l}\left(\begin{array}{c}
l \\
k^{\prime}
\end{array}\right) \frac{(-1)^{\left(k^{\prime}+k\right)}\left(k^{\prime}+k-1\right) !}{(k-1) !} \frac{1}{t^{k+k^{\prime}}} \mathrm{D}_{t}^{l-k^{\prime}-\beta_{i, d}-k} y(t)\right) \\
& -\sum_{i=0}^{N} \frac{a_{i}}{a_{N}} \sum_{j=1}^{m}\left(\begin{array}{c}
m \\
j
\end{array}\right) c_{i, j} \sum_{k=0}^{m-j}\left(\begin{array}{c}
m-j \\
k
\end{array}\right) \frac{\Gamma\left(\beta_{i, d}+j+k\right)}{\Gamma\left(\beta_{i, d}+j\right)} \times \\
& \sum_{k^{\prime}=0}^{l}\left(\begin{array}{c}
l \\
k^{\prime}
\end{array}\right) \frac{(-1)^{\left(j+k+k^{\prime}\right)}\left(k^{\prime}+k+j-1\right) !}{(k+j-1) ! t^{k+j+k^{\prime}}} \mathrm{D}_{t}^{l-k^{\prime}-\beta_{i, d}-j-k} y(t),
\end{aligned}
$$

$$
\begin{aligned}
& \frac{d^{l}}{d t^{l}} \int_{0}^{t} Q_{d, m}(t, \tau) u(\tau) d \tau=\frac{1}{a_{N}} \mathrm{D}_{t}^{l-\beta_{0, d}} u(t) \\
& +\frac{1}{a_{N}} \sum_{k=1}^{m}\left(\begin{array}{c}
m \\
k
\end{array}\right) \frac{\Gamma\left(\beta_{0, d}+k\right)}{\Gamma\left(\beta_{0, d}\right)} \times \\
& \sum_{k^{\prime}=0}^{l}\left(\begin{array}{c}
l \\
k^{\prime}
\end{array}\right) \frac{(-1)^{\left(k^{\prime}+k\right)}\left(k^{\prime}+k-1\right) !}{(k-1) ! t^{k+k^{\prime}}} \mathrm{D}_{t}^{l-k^{\prime}-\beta_{0, d}-k} u(t),
\end{aligned}
$$

$N_{\beta_{d}}=N-1$ if $\beta_{d}=0, N_{\beta_{d}}=N$ else, and $c_{i, j}$ are the coefficients of $\left(s^{\alpha_{i}}\right)^{(j)}$, which are given by (11).

Proof. (27) can be obtained using (6) and Theorem 1. Finally, (28)-(29) can be obtained by applying the classical Leibniz formula, where two cases of $k=0$ and $k \neq 0$ are considered in (29).

Remark 3 If $q=1$ in (12), the defined systems are integer order ones. On the one hand, in the case of $\beta_{q} \neq 0$, Corollary 1 permits to calculate the non-integer order 
derivatives of the output of an integer order system, as done in [14]. Thus, this paper is an extension of [14]. On the other hand, in the case of $\beta_{q}=0$, Corollary 1 permits to calculate the integer order derivatives of the output of an integer order system, i.e. it is a model-based integer order differentiator. Thus, this paper is also an extension of [22].

Similar to Theorem $1, \forall \nu_{d} \in \mathbb{R}_{+}^{*}, \mathrm{D}_{t}^{\nu_{d}} y$ is exactly given by proper integrals of $y$ and a part involving $u$ using a recursive way. Indeed, $\mathrm{D}_{t}^{\nu_{d}} y$ is obtained by taking the integer order derivative of $\mathrm{D}_{t}^{-\beta_{d}} y$ and calculated by (27)(29). Using Tab. 1 and Tab. 2, the required orders of the fractional integrals and derivatives of $u$ and $y$ are given in Tab. 3 and Tab. 4 , respectively, for $d=1, \ldots, q$, where $l=1$ in Tab. 4.

\begin{tabular}{|c|c|}
\hline \hline $\mathrm{D}_{t}^{\nu_{d}} y$ & $\mathrm{D}_{t}^{l-\beta_{0, d}} u$ and $\mathrm{D}_{t}^{l-k^{\prime}-\beta_{0, d}-k} u$ \\
\hline$-\nu_{1}=\beta_{1}-l$ & $\left\{\alpha_{N}-\nu_{1}, \ldots, \alpha_{N}+\beta_{1}+m\right\}$ \\
\hline$\vdots$ & $\vdots$ \\
\hline$-\nu_{d}=\beta_{d}-l$ & $\left\{\alpha_{N}-\nu_{d}, \ldots, \alpha_{N}+\beta_{d}+m\right\}$ \\
\hline$\vdots$ & $\vdots$ \\
\hline$-\nu_{q}=\beta_{q}-l$ & $\left\{\alpha_{N}-\nu_{q}, \ldots, \alpha_{N}+\beta_{q}+m\right\}$ \\
\hline \hline
\end{tabular}

Table 3

Required orders of the fractional integrals and derivatives of $u$ in the formula of $\mathrm{D}_{t}^{\nu_{d}} y$.

\begin{tabular}{|c|c|}
\hline \hline $\mathrm{D}_{t}^{\nu_{d}} y$ & $\mathrm{D}_{t}^{1-\beta_{i, d}} y$ \\
\hline$-\nu_{1}=\beta_{q}-\frac{1}{q}$ & $\left\{\beta_{q}, \beta_{q}+\frac{1}{q}, \ldots, \beta_{1}, \ldots, \alpha_{N}-\nu_{1}\right\}$ \\
\hline$\vdots$ & $\vdots$ \\
\hline$-\nu_{d}=\beta_{q}-\frac{d}{q}$ & $\left\{\alpha_{1}-\nu_{d}=-\nu_{d-1}, \ldots,-\nu_{1}, \ldots, \alpha_{N}-\nu_{d}\right\}$ \\
\hline$\vdots$ & $\vdots$ \\
\hline$-\nu_{q}=\beta_{q}-1$ & $\left\{\alpha_{1}-\nu_{q}, \ldots,-\nu_{1}, \beta_{q} \ldots, \alpha_{N}-\nu_{q}\right\}$ \\
\hline $\mathrm{D}_{t}^{\nu_{d}} y$ & $\mathrm{D}_{t}^{1-k^{\prime}-\beta_{i, d}-k} y$ and $\mathrm{D}_{t}^{1-k^{\prime}-\beta_{i, d}-j-k} y$ \\
\hline$-\nu_{1}=\beta_{q}-\frac{1}{q}$ & $\left\{\alpha_{1}-\nu_{1}+1, \ldots, \alpha_{N}+\beta_{1}+m\right\}$ \\
\hline$\vdots$ & $\vdots$ \\
\hline$-\nu_{d}=\beta_{q}-\frac{d}{q}$ & $\left\{\alpha_{1}-\nu_{d}+1, \ldots, \alpha_{N}+\beta_{d}+m\right\}$ \\
\hline$\vdots$ & $\vdots$ \\
\hline$-\nu_{q}=\beta_{q}-1$ & $\left\{\alpha_{1}-\nu_{q}+1 \ldots, \alpha_{N}+\beta_{q}+m\right\}$ \\
\hline \hline
\end{tabular}

\section{Table 4}

Required orders of the fractional integrals and derivatives of $y$ in the formula of $\mathrm{D}_{t}^{\nu_{d}} y$ in the case where $l=1$.

Then, on the one hand, we have: $\forall \nu_{d} \in \mathbb{R}_{+}^{*}, \mathrm{D}_{t}^{\nu_{d}} y$ involves $\mathrm{D}_{t}^{l-\beta_{0, d}} u$ and $\mathrm{D}_{t}^{l-k^{\prime}-\beta_{0, d}-k} u$, whose orders vary from $\alpha_{N}-\nu_{d}$ to $\alpha_{N}+\beta_{d}+m$. If $\alpha_{N}-\nu_{d} \geq 1$, all the integrals of $u$ in $\mathrm{D}_{t}^{\nu_{d}} y$ are proper. If $0<\alpha_{N}-\nu_{d}<1$, $\mathrm{D}_{t}^{\nu_{d}} y$ contains improper integrals of $u$. In the two previous cases, if the input $u$ contains noises, the noise effect can be reduced by the integrals considered as low-pass filters. If $\alpha_{N}-\nu_{d}=0, \mathrm{D}_{t}^{\nu_{d}} y$ contains $u$. If $\alpha_{N}-\nu_{d}<0$, $\mathrm{D}_{t}^{\nu_{d}} y$ contains the fractional derivatives of $u$.

On the other hand, we have:

- for $l=1, d=1, \mathrm{D}_{t}^{\nu_{1}} y$ contains $(i) \mathrm{D}_{t}^{-\beta_{b}} y$ for $b=$ $1, \ldots, q$, with $0 \leq \beta_{b}<1$, which are given by proper integrals of $y$ by Theorem $1,(i i)$ the fractional integrals of $y$ with orders varying from 1 to $\alpha_{N}+\beta_{1}+m$, which are proper;

- for $l=1, d \in\{2, \ldots, q\}, \mathrm{D}_{t}^{\nu_{d}} y$ contains $(i)$ the fractional derivatives $\mathrm{D}_{t}^{\nu_{b}} y$ for $b=1, \ldots, d-1$, which are previously given by proper integrals of $y$ in Corollary 1 , (ii) $\mathrm{D}_{t}^{-\beta_{b}} y$ for $b=1, \ldots, q$, with $0 \leq \beta_{b}<1$, which are given by Theorem 1, (iii) the fractional integrals of $y$ with orders varying from 1 to $\alpha_{N}+\beta_{d}+m$;

- similarly, for $\nu_{d}>1, \mathrm{D}_{t}^{\nu_{d}} y$ contains $(i)$ the fractional derivatives $\mathrm{D}_{t}^{\alpha} y$ for $\alpha=\nu_{d}-\frac{1}{q}, \nu_{d}-\frac{2}{q}, \ldots, \frac{1}{q}-\beta_{q}>0$, which are previously given by Corollary 1 , (ii) $\mathrm{D}_{t}^{-\beta_{b}} y$ for $b=1, \ldots, q$, with $0 \leq \beta_{b}<1$, which are given in Theorem 1, (iii) the fractional integrals of $y$ with orders varying from 1 to $\alpha_{N}+\beta_{d}+m$.

According to the previous analysis, $\mathrm{D}_{t}^{\nu_{d}} y$ is given using a recursive way, which only contains proper integrals of $y$ and a part involving $u$. By grouping the integrals of $y$, $\mathrm{D}_{t}^{\nu_{d}} y$ can be given by an algebraic formula as follows:

$$
\mathrm{D}_{t}^{\nu_{d}} y(t)=\int_{0}^{t} W_{m}(\tau) y(\tau) d \tau+U_{m}(t),
$$

where $U_{m}$ denotes the part involving $u$, and $W_{m}$ denotes the corresponding function in the integral of $y$, which is the sum of the associated functions in the proper integrals of $y$ in $\mathrm{D}_{t}^{\alpha} y$ for $\alpha \in\left\{\nu_{d}-\frac{1}{q}, \ldots, \frac{1}{q}-\right.$ $\left.\beta_{q},-\beta_{q}, \ldots,-\beta_{1},-1, \ldots,-\alpha_{N}-\beta_{d}-m\right\}$. Hence, $W_{m}$ is bounded on $[0, t]$.

Consequently, the Riemann-Liouville fractional derivative of $y$ of an arbitrary order is exactly given by a new algebraic formula in Corollary 1, i.e. it does not contain any source of errors in continuous noise-free case, and can provide non-asymptotic estimations in finite-time without knowing the initial conditions of the considered system. Moreover, the proper integrals of $y$ can reduce the noise error contribution if the noisy observation of $y$ is used. In the next subsection, a digital fractional order differentiator is introduced in discrete noisy cases, where some error analysis is also given based on (30).

\subsection{Digital fractional order differentiator in discrete noisy cases}

From now on, let $y^{\varpi}$ be a discrete noisy observation of $y$ on $I=[0, h] \subset \mathbb{R}_{+}$:

$$
y^{\varpi}\left(t_{i}\right)=y\left(t_{i}\right)+\varpi\left(t_{i}\right),
$$


where $t_{i}=i T_{e}$, for $i=0,1, \ldots, M$, with an equidistant sampling period $T_{e}=\frac{h}{M}$. Moreover, the noise $\{\varpi(t), t \in$ $I\}$ is assumed to be a continuous stochastic process satisfying the following conditions:

$\left(C_{1}\right)$ : for any $t_{1}, t_{2} \in I, t_{1} \neq t_{2}, \varpi\left(t_{1}\right)$ and $\varpi\left(t_{2}\right)$ are independent;

$\left(C_{2}\right)$ : the mean value function of $\{\varpi(t), t \in I\}$ denoted by $\mathbb{E}[\cdot]$ is equal to zero;

$\left(C_{3}\right)$ : the variance function of $\{\varpi(t), t \in I\}$ denoted by $\operatorname{Var}[\cdot]$ is bounded on $I$, i.e. $\exists \delta \in \mathbb{R}_{+}, \forall t \in$ $I, \operatorname{Var}[\varpi(t)] \leq \delta$.

Note that a zero-mean white Gaussian noise satisfies these conditions.

The Riemann-Liouville fractional integrals and derivatives of the output $y$ can be numerically approximated by the following Grünwald-Letnikov scheme $[1,5]: \forall \alpha \in \mathbb{R}$,

$$
\mathrm{D}_{t}^{\alpha} y(t) \approx \frac{1}{T_{e}^{\alpha}} \sum_{j=0}^{\left\lceil\frac{t}{T_{e}}\right\rceil} w_{j}^{(\alpha)} y\left(t-j T_{e}\right),
$$

where the binomial coefficients can be recursively calculated as follows:

$$
\begin{cases}w_{0}^{(\alpha)}=1, & \text { if } j=0 \\ w_{j}^{(\alpha)}=\left(1-\frac{\alpha+1}{j}\right) w_{j-1}^{(\alpha)}, & \text { else. }\end{cases}
$$

However, this scheme is not robust against noises.

Different from the Grünwald-Letnikov scheme, according to (30), the formula of $\mathrm{D}_{t}^{\nu_{d}} y$ contains two parts: the one given by a proper integral of $y$, and the other one involving $u$. In order to estimate the Riemann-Liouville fractional derivatives of $y$ in discrete noisy cases, $\mathrm{D}_{t}^{\nu_{d}} y$ is approximated by the following digital fractional order differentiator: for $i=1, \ldots, M$,

$$
D_{m}^{\nu_{d}} y^{\varpi}\left(t_{i}\right):=T_{e} \sum_{j=0}^{i} w_{j} W_{m}\left(t_{j}\right) y^{\varpi}\left(t_{j}\right)+\tilde{U}_{m}\left(t_{i}\right),
$$

where $w_{i} \in \mathbb{R}_{+}$are the weights of a given numerical integration method. According to previous analysis, $\tilde{U}_{m}\left(t_{i}\right)$ is calculated in the following way:

- the fractional integrals of $u$ of order larger than 1 are calculated using a numerical integration method;

- the fractional integrals of $u$ of order strictly smaller than 1 and the fractional derivatives of $u$ are numerically calculated using the Grünwald-Letnikov scheme given in (32)-(33).

Consequently, the digital fractional order differentiator $D_{m}^{\nu_{d}} y^{\varpi}\left(t_{i}\right)$ can be used to estimate the RiemannLiouville fractional derivative $\mathrm{D}_{t}^{\nu_{d}} y$ for any $\nu_{d} \in \mathbb{R}_{+}^{*}$, where $y$ is the output of a fractional order linear system defined in (12). It contains three sources of errors:

- the numerical error due to the numerical integration method used to approximate the proper integral involving $y$;

- the noise error contribution of the following form:

$$
e_{m}^{\varpi}\left(t_{i}\right):=T_{e} \sum_{j=0}^{i} w_{j} W_{m}\left(t_{j}\right) \varpi\left(t_{j}\right) ;
$$

- the numerical errors in $\tilde{U}_{m}\left(t_{i}\right)$, which are due to the numerical integration method and the Grünwald-Letnikov scheme, respectively.

Firstly, it is well known that the numerical errors due to the numerical integration method converge to zero when $T_{e} \rightarrow 0$ [24]. Secondly, as shown previously, the Grünwald-Letnikov scheme is used to approximate $\mathrm{D}_{t}^{\alpha} u$ with $-1<\alpha$ in the calculation of $\tilde{U}_{m}\left(t_{i}\right)$. It is shown in [4] that in the case of $-1<\alpha<0$ (resp. $\alpha>0$ ), if $u$ is continuous on $I$ (resp. $u$ is $\lceil\alpha\rceil$-times continuously differentiable on $I$ ), the produced numerical error converges to zero when $T_{e} \rightarrow 0$. Thirdly, if the noise satisfies conditions $\left(C_{1}\right)-\left(C_{3}\right)$, it can be shown that the noise error contribution $e_{m}^{\varpi}\left(t_{i}\right)$ converges to zero in mean square when $T_{e} \rightarrow 0$ (see [14] for more details). A similar result can also be obtained using non-standard analysis as done in [18]. Consequently, both the numerical errors and the noise error contribution can be reduced in the proposed digital fractional order differentiator by decreasing the sampling period $T_{e}$. However, if the computations are performed on a finite precision numerical machine, there are also round-off errors [25]. Hence, the infinite reduction of $T_{e}$ would not lead to arbitrary reduction of estimation errors, since the round-off errors would become too large at some points.

When the sampling period is set, by applying $\left(C_{1}\right)-\left(C_{3}\right)$ and the properties of the mean value and the variance functions to (35), one obtains:

$$
\begin{aligned}
\mathbb{E}\left[e_{m}^{\varpi}\left(t_{i}\right)\right] & =0 \\
\operatorname{Var}\left[e_{m}^{\varpi}\left(t_{i}\right)\right] & =T_{e}^{2} \sum_{j=0}^{i} w_{j}^{2} W_{m}^{2}\left(t_{j}\right) \operatorname{Var}\left[\varpi\left(t_{j}\right)\right] \\
& \leq \delta T_{e}^{2} \sum_{j=0}^{i} w_{j}^{2} W_{m}^{2}\left(t_{j}\right) .
\end{aligned}
$$

Hence, the noise error contribution $e_{m}^{\varpi}\left(t_{i}\right)$ can be bounded using the Bienaymé-Chebyshev inequality:

$$
\forall \gamma \in \mathbb{R}_{+}^{*}, \operatorname{Pr}\left(\left|e_{m}^{\varpi}\left(t_{i}\right)\right|<\gamma\left(\operatorname{Var}\left[e_{m}^{\varpi}\left(t_{i}\right)\right]\right)^{\frac{1}{2}}\right)>1-\frac{1}{\gamma^{2}}
$$


i.e. the probability for $\left|e_{m}^{\varpi}\left(t_{i}\right)\right|$ to be smaller than $\gamma\left(\operatorname{Var}\left[e_{m}^{\varpi}\left(t_{i}\right)\right]\right)^{\frac{1}{2}}$ is larger than $1-\frac{1}{\gamma^{2}}$. Thus, the following error bound is deduced from (37) and (38):

$$
\left|e_{m}^{\varpi}\left(t_{i}\right)\right| \stackrel{p_{\gamma}}{<} \gamma T_{e}\left(\delta \sum_{j=0}^{i} w_{j}^{2} W_{m}^{2}\left(t_{j}\right)\right)^{\frac{1}{2}}
$$

where $a \stackrel{p_{\gamma}}{<} b$ means that the probability for a real number $b$ to be larger than another real number $a$ is equal to $p_{\gamma}$ with $1-\frac{1}{\gamma^{2}}<p_{\gamma}<1$. Remark that the value of $p_{\gamma}$ can be given by the probability density function of the considered noise $\varpi$. In particular, if $\varpi$ is a zero-mean white Gaussian noise, it can be deduced that $e_{m}^{\varpi}\left(t_{i}\right)$ is a normally distributed random number. Thus, according to the three-sigma rule, we have: $p_{1}=68.26 \%, p_{2}=$ $95.44 \%$ and $p_{3}=99.73 \%$, for $\gamma=1,2,3$, respectively.

Thanks to the probability properties of the mean value and the variance functions, and the BienayméChebyshev inequality, the noise error bound obtained in (39) is sharp, especially in white Gaussian noise cases. Since it depends on the design parameter $m$, the study of the influence of $m$ on this error bound is useful to deduce the influence of $m$ on the noise error contribution. Consequently, the noise error contribution can be reduced by choosing the value of $m$ which minimizes the noise error bound.

Finally, according to the previous analysis, the following algorithm is required to realize the proposed differentiator $D_{m}^{\nu_{d}} y^{\varpi}\left(t_{i}\right)$, which is devoted to applying Theorem 1 , Corollary 1 , and contains the following steps:

Step 1. find $\beta_{q} \in\left[0, \frac{1}{q}\left[\right.\right.$ such that $\nu_{d}=\left\lceil\nu_{d}\right\rceil-1+\frac{d}{q}-\beta_{q}$ with $d \in\{1, \ldots, q\}$;

Step 2. using $\beta_{q}$, calculate $\beta_{j}$ for $j=1, \ldots, q-1$;

Step 3. using (13)-(15), calculate $\mathrm{D}_{t}^{-\beta_{1}} y$, where all the integrals $\mathrm{D}_{t}^{-\alpha} y$ and $\mathrm{D}_{t}^{-\alpha} u$ are proper with $\alpha \geq 1$, and calculated using a numerical integration method;

Step 4. using (13)-(15), calculate $\mathrm{D}_{t}^{-\beta_{j}} y$ for $j=$ $2, \ldots, q$ in a loop. For each $j$, the fractional integrals $\mathrm{D}_{t}^{-\alpha} y\left(\right.$ resp. $\left.\mathrm{D}_{t}^{-\alpha} u\right)$ in the expression of $\mathrm{D}_{t}^{-\beta_{j}} y$ are calculated as follows:

- if $\alpha \geq 1$, they are calculated using the numerical integration method,

- otherwise they are previously calculated (resp. calculated using the Grünwald-Letnikov scheme);

Step 5. using (27)-(29), calculate $\mathrm{D}_{t}^{\alpha_{i}} y$ with $\alpha_{i}=\nu_{d}-$ $\frac{i}{q}$, for $i=\left(\nu_{d}+\beta_{q}\right) q-1, \ldots, 0$ in a loop. For each $i$, the fractional integrals and derivatives $\mathrm{D}_{t}^{-\alpha} y$ and $\mathrm{D}_{t}^{-\alpha} u$ in the expression of $\mathrm{D}_{t}^{\alpha_{i}} y$ are calculated using a similar way as done in Step 2 .

\section{Simulation results}

In order to show the accuracy and the robustness of the proposed fractional differentiator, some numerical results are presented in this section.

Example 1: In most cases, neither the fractional derivatives of a function nor the solution of a fractional order differential equation can be analytically calculated. In order to obtain the exact value of the sought fractional derivative, the following academic model is considered in this example: $\forall t \in] 0,30]$,

$$
\sum_{i=0}^{4} a_{i} \mathrm{D}_{t}^{\frac{i}{3}} y(t)=\sum_{i=0}^{4} a_{i} \mathrm{D}_{t}^{\frac{i}{3}} u(t),
$$

where $a_{i}=1$ for $i=0, \ldots, 4, u(t)=y(t)=y_{1}(t)+y_{2}(t)$, $y_{1}(t)=\sin \left(\omega_{1} t\right)$ and $y_{2}(t)=\cos \left(\omega_{2} t\right)$ with $\omega_{1}=1.2$ and $\omega_{2}=0.6$. The analytical Riemann-Liouville fractional derivatives of $y_{1}$ and $y_{2}$ can be found in [2].

$\mathrm{D}_{t}^{\frac{1}{2}} y$ will be estimated in the discrete noisy case with $t_{i}=i T_{e} \in I=[0,30], T_{e}=0.01$, for $i=0, \ldots, 3000$, and $y^{\varpi}\left(t_{i}\right)=y\left(t_{i}\right)+\delta \varpi\left(t_{i}\right)$, where the noise $\left\{\varpi\left(t_{i}\right)\right\}$ is simulated from a zero-mean white Gaussian iid sequence, and the value of $\delta$ is adjusted such that the signal-tonoise ratio $\mathrm{SNR}=10 \log _{10}\left(\frac{\sum\left|y^{\varpi}\left(t_{i}\right)\right|^{2}}{\sum\left|\delta \varpi\left(t_{i}\right)\right|^{2}}\right)$ is equal to $\mathrm{SNR}=15 \mathrm{~dB}$ (see [26] for this well known concept in signal processing). The original output $y$ and the discrete noisy output $y^{\varpi}$ are shown in Fig. 1 . Then, the fractional order differentiator obtained in (34) is applied, where the trapezoidal numerical integration method is considered.

Firstly, it is shown how to choose the value of the design parameter $m$ using the noise error bound obtained in (39) with $\gamma=2$. Since the value of $\nu_{d}$ is set in Corollary 1 with $\nu_{d}=\frac{1}{2}$ and $\beta_{q}=\frac{2}{3}-\frac{1}{2}$, the expression of $\mathrm{D}_{t}^{\frac{1}{2}} y$ can be obtained. Thus, the expression of $W_{m}$ can be deduced in (30). Then, by taking different values of $m$, the values of the error bound obtained in (39) can be calculated at each $t_{i}$ for $i=0, \ldots, 3000$. Using such a way, the variation with respect to $m$ of the noise error bound can be observed in Fig. 2. According to Fig. 2, the influence of $m$ on the noise error contribution can be deduced. Hence, in order to reduce the noise error contribution, the following values of $m$ are chosen: $m=2$ for $t_{i} \in\left[2 T_{e}, 1.95\right], m=4$ for $t_{i} \in\left[1.95+T_{e}, 3.7\right], m=8$ for $t_{i} \in\left[3.7+T_{e}, 5.5\right], m=10$ for $t_{i} \in\left[5.5+T_{e}, 7.2\right]$, and $m=14$ for $t_{i} \in\left[7.2+T_{e}, 30\right]$.

Secondly, in order to compare with the proposed method the fractional order Legendre differentiator proposed in [13] is applied. The latter is a model-free differentiator and obtained by a polynomial approximation method. Hence, it contains a truncated term error which can be 


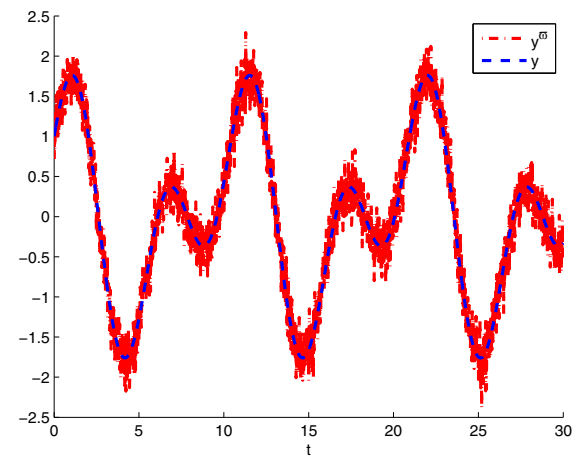

Fig. 1. Example 1. The output and its discrete noisy observation.

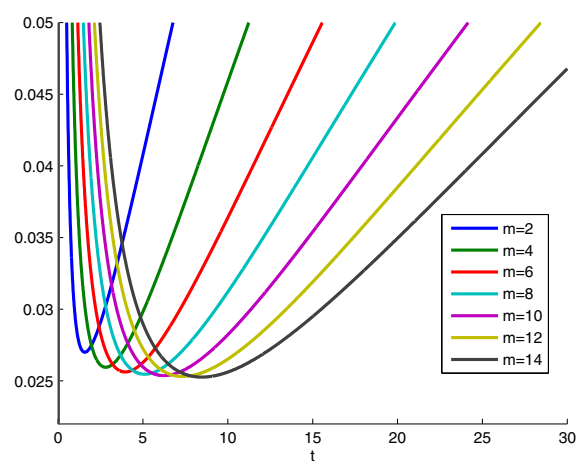

Fig. 2. Example 1. The variation of the noise error bound given in (39) with $m=2, \ldots, 14$.

significantly reduced by admitting a time-delay. Consequently, this differentiator depends on two design parameters: the order of the used polynomial $N$ and the timedelay $\vartheta$. According to the analysis done in [13], the design parameters are set in this example as follows: $\vartheta=0.8$, $N=6$ for $t_{i} \in[0.9,8], N=12$ for $t_{i} \in\left[8+T_{e}, 18\right]$, and $N=20$ for $t_{i} \in\left[18+T_{e}, 30\right]$.

The obtained estimations of $\mathrm{D}_{t}^{\frac{1}{2}} y$ are shown in Fig. 3. It can be seen that different from the fractional order Legendre differentiator the proposed differentiator does not produce a time-delay. The reason is that the proposed method is based on the system model. Finally, the corresponding absolute estimation errors are shown in Fig. 4, where the one for the fractional order Legendre differentiator is obtained by shifting the estimation to avoid the time-delay. The latter principally contains the amplitude error and the noise error contribution.

Example 2: In this example, a physical model is considered, which is introduced by a fractional order differential equation [27]. Let us consider a rigid plate immersed in a Newtonian fluid of infinite extent and connected by a massless spring to a fixed point. If a force is applied to the plate, the dynamic of the plate can be represented by

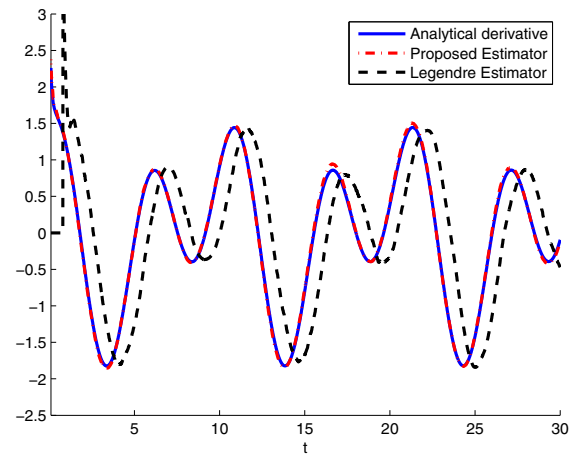

Fig. 3. Example 1. The exact value of $D_{t}^{\frac{1}{2}} y$ and its estimations obtained by the proposed method and the fractional order Legendre differentiator.

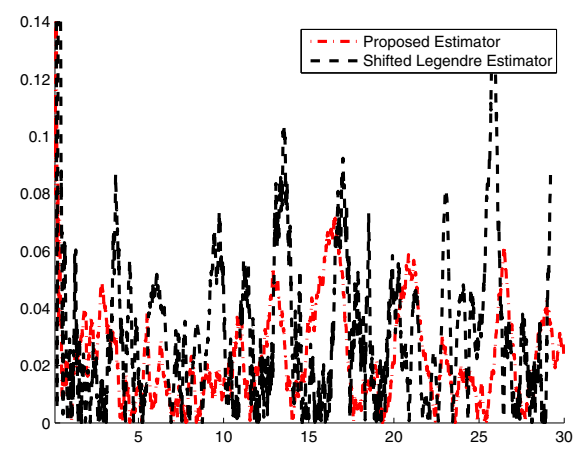

Fig. 4. Example 1. The estimation errors of $\mathrm{D}_{t}^{\frac{1}{2}} y$ produced by the proposed method and the shifted fractional order Legendre differentiator.

the following fractional order differential equation [27]:

$$
\forall t>0, \sum_{i=0}^{4} a_{i} \mathrm{D}_{t}^{\frac{i}{2}} y(t)=u(t),
$$

where $a_{4}=M_{p}, a_{3}=2 S \sqrt{\mu \rho}, a_{2}=a_{1}=0, a_{0}=K, M_{p}$ is the mass of the plate, $S$ is the area of the plate, $\mu$ is the fluid viscosity, $\rho$ is the fluid density, $K$ is the stiffness of the spring, $y$ is the displacement of the plate, and $u$ is the force. Moreover, this plate-fluid system is assumed to be initially in an equilibrium state with $y(0)=0$ and $\dot{y}(0)=0$.

The Riemann-Liouville fractional derivatives of the output $y$ will be estimated in the following situation with $t_{i}=i T_{e} \in I=[0,45], T_{e}=0.01$, for $i=0, \ldots, 4500$ :

- the output $y_{b i a s}$ is generated by the GrünwaldLetnikov scheme given in (32)-(33) from the following model: $a_{4} \mathrm{D}_{t_{i}}^{2} y\left(t_{i}\right)+a_{3} \mathrm{D}_{t_{i}}^{\frac{3}{2}} y\left(t_{i}\right)+a_{0} y\left(t_{i}\right)+\varpi_{d}\left(t_{i}\right)=u\left(t_{i}\right)$, where $a_{4}=1, a_{3}=a_{0}=0.5, u\left(t_{i}\right)=1$ for $0 \leq t_{i} \leq 1$, $u\left(t_{i}\right)=0$ for $t_{i}>1$, and $\varpi_{d}\left(t_{i}\right)=0.5 \sin \left(5 t_{i}\right)$ is a disturbance which biases the output; 


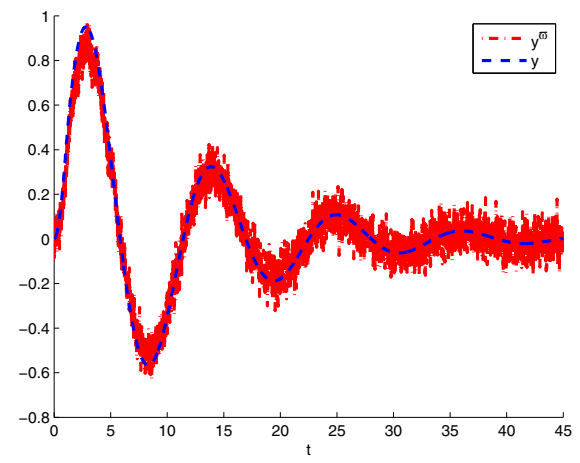

Fig. 5. Example 2. The output and its discrete noisy observation.

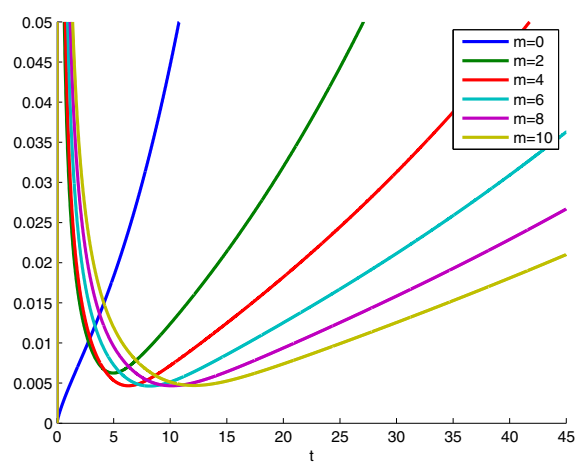

Fig. 6. Example 2. The variation of the noise error bound given in (39) with $m=0,2, \ldots, 10$.

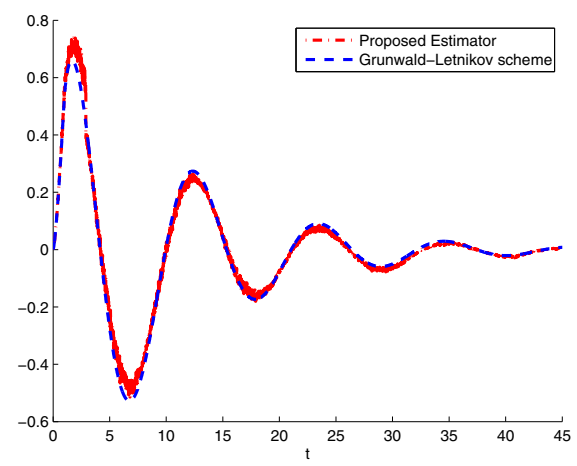

Fig. 7. Example 2. The numerical calculation and the estimation of $\mathrm{D}_{t}^{\frac{1}{2}} y$ obtained by the Grünwald-Letnikov scheme in discrete noise-free case and the proposed method in the discrete noisy case, respectively.

- the parameters $a_{j}$ for $j=0,3,4$, are known with errors: $\tilde{a}_{j}\left(t_{i}\right)=a_{j}+\varpi_{j}\left(t_{i}\right)$, where $\left\{\varpi_{j}\left(t_{i}\right)\right\}$ are simulated from the uniform $i i d$ sequences with $\left.\varpi_{j}\left(t_{i}\right) \in\right]-\delta_{j}, \delta_{j}[$ and $\delta_{j}=\frac{1}{20} a_{j}$;

- the input and output are corrupted by a zero-mean white Gaussian noise with SNR $=15 \mathrm{~dB}: u^{\varpi}\left(t_{i}\right)=$ $u\left(t_{i}\right)+\delta_{u} \varpi_{u}\left(t_{i}\right)$ and $y^{\varpi}\left(t_{i}\right)=y_{\text {bias }}\left(t_{i}\right)+\delta_{y} \varpi_{y}\left(t_{i}\right)$.

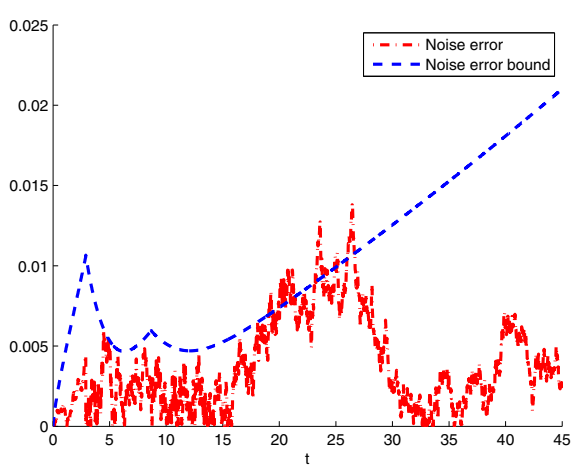

Fig. 8. Example 2. The error due to the noisy output in the estimation of $\mathrm{D}_{t}^{\frac{1}{2}} y$ obtained by the proposed method and the corresponding error bound.

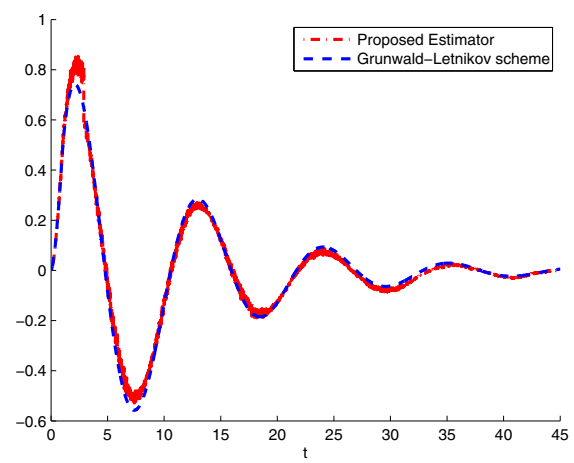

Fig. 9. Example 2. The numerical calculation and the estimation of $D_{t}^{0.3} y$ obtained by the Grünwald-Letnikov scheme in discrete noise-free case and the proposed method in the discrete noisy case, respectively.

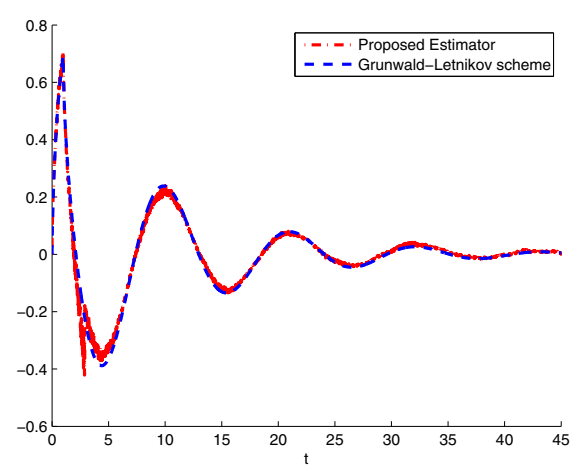

Fig. 10. Example 2. The numerical calculation and the estimation of $\mathrm{D}_{t}^{1.3} y$ obtained by the Grünwald-Letnikov scheme in discrete noise-free case and the proposed method in the discrete noisy case, respectively. 
The original output $y$ and the discrete noisy output $y^{\varpi}$ are shown in Fig. 5.

Firstly, using a similar way as done in Example 1, the values of the design parameter $m$ are set. For this purpose, $\mathrm{D}_{t}^{\frac{1}{2}} y$ is considered as an example. Then, the values of the corresponding error bound are calculated at each $t_{i}$ for $i=0, \ldots, 4500$, and the variation with respect to $m$ is shown in Fig. 6, where the values of the parameters $a_{j}$ for $j=0,3,4$, are used. Hence, according to Fig. 6, the following values of $m$ are chosen: $m=0$ for $t_{i} \in\left[T_{e}, 2.9\right], m=4$ for $t_{i} \in\left[2.9+T_{e}, 8.7\right]$, and $m=10$ for $t_{i} \in\left[8.7+T_{e}, 45\right]$.

Secondly, since the analytical Riemann-Liouville fractional derivatives of the output are unknown, the Grünwald-Letnikov scheme is applied in the noise-free case to verify the efficiency of the proposed fractional order differentiator. The obtained estimation of $\mathrm{D}_{t}^{\frac{1}{2}} y$ is shown in Fig. 7. It can be seen that the proposed method is robust. Hence, it is interesting to provide some robustness analysis on the proposed method in the considered example.

- By taking $\delta_{y} \varpi_{y}$ and the expression of $W_{m}$ in (35), the error due to the noisy output can be calculated at each $t_{i}$ using different values of $m$ chosen previously. Moreover, the corresponding noise error bound can also be calculated using (39) with $\gamma=2$. The obtained results are shown in Fig. 8, where it can be seen that when the time $t$ becomes large, the noise error bound is increasing with $t$. Hence, the noise error can also be increasing with time. In order to solve this problem, on the one hand, according to Fig. 6, the value of $m$ should be increased to reduce the noise error bound; on the other hand, according to the analysis done in Subsection 3.3, the value of sampling period $T_{e}$ should be reduced.

- According to the analysis done in Subsection 3.2, the expression of $\mathrm{D}_{t}^{\nu_{d}} y$ contains a proper integral of $y$ and a part involving $u$ in (30). Moreover, if $\nu_{d}<\alpha_{N}$, the part involving $u$ is an integral of $u$, either proper or improper. In this example, $\alpha_{N}-\nu_{d}=2-\frac{1}{2}=\frac{3}{2}>1$, the part involving $u$ is a proper integral of $u$. This is why the noise effect in the input is reduced in the estimation of $\mathrm{D}_{t}^{\frac{1}{2}} y$, as the one in the output.

- Assume $\tilde{a}_{j}=a_{j}+\varpi_{j}$ for $j=0, \ldots, N$, with $\left|\varpi_{j}\right| \leq$ $\delta_{j}<\left|a_{j}\right|$. It can be seen in (27)-(29) that the formula of $\mathrm{D}_{t}^{\nu_{d}} y$ is linear with respect to $\frac{1}{a_{N}}$ and $\frac{a_{j}}{a_{N}}$. Consequently, the error due to $\varpi_{j}$ in $\mathrm{D}_{t}^{\nu_{d}} y$ depends on the relative errors in $\frac{1}{a_{N}}$ and $\frac{a_{j}}{a_{N}}$, which can be bounded as follows:

$$
\left|a_{N}\right|\left|\frac{1}{\tilde{a}_{N}}-\frac{1}{a_{N}}\right|=\left|\frac{-\varpi_{N}}{a_{N}+\varpi_{N}}\right| \leq \frac{\delta_{N}}{\left|a_{N}\right|-\delta_{N}},
$$

$$
\begin{aligned}
\left|\frac{a_{N}}{a_{j}}\right|\left|\frac{\tilde{a}_{j}}{\tilde{a}_{N}}-\frac{a_{j}}{a_{N}}\right| & =\left|\frac{a_{N} \varpi_{j}-a_{j} \varpi_{N}}{a_{j}\left(a_{N}+\varpi_{N}\right)}\right| \\
& \leq \frac{\left|a_{N}\right| \delta_{j}+\left|a_{j}\right| \delta_{N}}{\left|a_{j}\right|\left(\left|a_{N}\right|-\delta_{N}\right)} .
\end{aligned}
$$

Hence, these relative errors are increasing with respect to $\delta_{j}$. In this example, $\delta_{j}=\frac{1}{20} a_{j}$ is chosen. This is why the obtained estimation is robust. Although there are multiplicative noises in the estimation due to the noises in the output and the input, since it is shown previously that the noise effect in the output and the input can be significantly reduced, the error due to $\varpi_{j}$ plays a more important role.

- If there is a disturbance in the model, it plays as a part of the input and produces a bias term to the output. Then, using (34) the error due to this bias term can be given as follows:

$$
e_{\text {bias }}\left(t_{i}\right):=T_{e} \sum_{j=0}^{i} w_{j} W_{m}\left(t_{j}\right)\left(y_{b i a s}\left(t_{j}\right)-y\left(t_{j}\right)\right)
$$

Hence, if the fractional derivative of the bias term $y_{\text {bias }}-$ $y$ exists, $e_{\text {bias }}$ is its estimation. As shown in Subsection 3.2, $W_{m}$ is bounded. If the bias term is also bounded, $e_{\text {bias }}$ is bounded. Thus, $e_{\text {bias }}$ depends on how the output is biased, which depends on the structure of the model and the source of the disturbance. In this example, the disturbance $\varpi_{d}\left(t_{i}\right)=0.5 \sin \left(5 t_{i}\right)$ does not significantly change the output. This is why the obtained estimation is robust.

Consequently, it can be concluded that the proposed method can well cope with the corrupting noises in the output (resp. in the input if $\nu_{d}<\alpha_{N}$ ). Moreover, if the errors in the model parameters and the bias term in the output are not relatively large, robust estimations can also be obtained by the proposed method.

Finally, $\mathrm{D}_{t}^{0.3} y$ and $\mathrm{D}_{t}^{1.3} y$ are estimated by taking $\beta_{q}=$ 0.2 in Corollary 1. The same values of $m$ are chosen as done in the estimation of $D_{t}^{\frac{1}{2}} y$. The obtained estimations are shown in Fig. 9 and Fig. 10, respectively.

\section{Conclusions}

In this paper, a non-asymptotic fractional order differentiator was introduced to estimate the Riemann-Liouville fractional derivatives of the output of a class of fractional order linear systems in discrete noisy environment. Firstly, by applying the algebraic method and the Leibniz formula for Riemann-Liouville fractional integrals, the fractional integral of order strictly smaller than 1 of the output was expressed by an integral formula. Then, a new algebraic formula for the fractional derivative of 
an arbitrary order was deduced by taking the integer order derivative of the obtained formula for fractional integrals. This formula does not contain any source of errors in continuous noise-free case. Hence, it can provide estimations in finite-time without knowing the initial conditions of the considered systems. Secondly, a digital fractional order differentiator was introduced in discrete noisy cases. Then, some error analysis was given, where an error bound useful for the selection of the design parameter was provided. Finally, numerical results were given to show the accuracy and the robustness of the proposed fractional order differentiator.

\section{References}

[1] Podlubny, I. (1998). Fractional differential equations: an introduction to fractional derivatives, fractional differential equations, to methods of their solution and some of their applications (Vol. 198). Academic press.

[2] Miller, K. S., \& Ross, B. (1993). An Introduction to the Fractional Calculus and Fractional Differential Equations. Wiley, New York.

[3] Kilbas, A. A., Srivastava, H. M., \& Trujillo, J. J. (2006). Theory and Applications of Fractional Differential Equations. vol. 204 of North-Holland Mathematics Studies, Elsevier, Amsterdam, The Netherlands.

[4] Diethelm, K. (2010). The analysis of fractional differential equations: An application-oriented exposition using differential operators of Caputo type. vol. 2004, Springer.

[5] Concepción, A. M., Chen, Y. Q., Vinagre, B. M., Xue, D. Y. , \& Feliu-Batlle, V. (2010). Fractional-order Systems and Controls: Fundamentals and Applications. Springer.

[6] Bandyopadhyay, B., \& Kamal, S. (2015). Stabilization and Control of Fractional Order Systems: A Sliding Mode Approach. Springer International Publishing.

[7] Yin, C., Chen, Y. Q., \& Zhong, S. M. (2014). Fractionalorder sliding mode based extremum seeking control of a class of nonlinear systems. Automatica, 50(12), 3173-3181.

[8] Victor, S., Malti, R., Garnier, H., \& Oustaloup, A. (2013). Parameter and differentiation order estimation in fractional models. Automatica, 49(4), 926-935.

[9] Sabatier, J., Farges, C., Merveillaut, M., \& Fenetau, L. (2012). On observability and pseudo state estimation of fractional order systems. European Journal of Control, 18(3), 260-271.

[10] Machado, J. A. T. (2009). Calculation of fractional derivatives of noisy data with genetic algorithms. Nonlinear Dynamics, 57, 253-260.

[11] Machado, J. A. T. (2012). Exploiting sensor redundancy for the calculation of fractional derivatives in the presence of noise. Signal Processing, 92, 204-209.

[12] Chen, D. L., Chen, Y. Q., \& Xue, D. Y. (2011). Digital Fractional Order Savitzky-Golay Differentiator. IEEE Transactions on Circuits \& Systems II: Express Briefs, 58(11), 758-762.

[13] Liu, D. Y., Gibaru, O., Perruquetti, W., \& Laleg-Kirati, T. M. (2015). Fractional order differentiation by integration and error analysis in noisy environment. IEEE Transactions on Automatic Control, 60(11), 2945-2960.
[14] Liu, D. Y., Tian, Y., Boutat, D., \& Laleg-Kirati, T. M. (2015). An algebraic fractional order differentiator for a class of signals satisfying a linear differential equation. Signal Processing, 116, 78-90.

[15] Liu, D. Y., \& Laleg-Kirati, T. M. (2015). Robust fractional order differentiators using generalized modulating functions method. Signal Processing, 107, 395-406.

[16] Wei, X., Liu, D. Y., \& Boutat, D. (2016). Non-Asymptotic Pseudo-State Estimation for a Class of Fractional Order Linear Systems. IEEE Transactions on Automatic Control, DOI: $10.1109 /$ TAC.2016.2575830.

[17] Fliess, M., \& Sira-Ramírez, H. (2003). An algebraic framework for linear identification. ESAIM Control Optim. Calc. Variat., 9, 151-168.

[18] Fliess, M. (2006). Analyse non standard du bruit. C.R. Acad. Sci. Paris, Ser. I, 342, 797-802.

[19] Mboup, M., Join, C., \& Fliess, M. (2009). Numerical differentiation with annihilators in noisy environment. Numerical Algorithms, 50(4), 439-467.

[20] Liu, D. Y., Gibaru, O., \& Perruquetti, W. (2011). Error analysis of Jacobi derivative estimators for noisy signals. Numerical Algorithms, 58(1), 53-83.

[21] Fliess, M., \& Sira-Ramírez, H. (2004). Reconstructeurs d'états. C.R. Acad. Sci. Paris, Série I, 338, 91-96.

[22] Tian, Y., Floquet, T., \& Perruquetti, W. (2008). Fast state estimation in linear time-varying systems: an algebraic approach. in Proc. 47th IEEE Conference on Decision \&f Control, Cancun Mexique.

[23] Abramowitz, M., \& Stegun, I.A., editeurs (1965). Handbook of mathematical functions. GPO.

[24] Ralston, A. (1965). A first course in numerical analysis. McGraw-Hill, New York.

[25] Butt, R. (2009). Introduction to Numerical Analysis Using MATLAB. Jones and Bartlett Learning.

[26] Haykin, S., \& Van Veen, B. (2002). Signals \& Systems. 2nd edn. John Wiley and Sons.

[27] Torvik, P. J. \& Bagley, R. L. (1984). On the appearance of the fractional derivative in the behavior of real materials. Journal of Applied Mechanics, 51, 294-298. 